DE

M E D I C I N A

T R O P I C A L

$\mathrm{DE}$

SÃO PAULO

JOURNAL OF THE SÃO PAULO INSTITUTE OF TROPICAL MEDICINE
${ }^{1}$ Universidade Federal de São João del-Rei, Divinópolis, Minas Gerais, Brazil

Correspondence to: Virgínia Paula Frade Universidade Federal de São João del-Rei, Campus Centro-Oeste Dona Lindu, Rua Sebastiao Gonçalves Coelho, 400, CEP 35501-296, Divinópolis, MG, Brazil Tel: + 553732211164

E-mail: virginiafrade20@yahoo.com.br

Received: 2 March 2020

Accepted: 1 July 2020

\section{Ideal benznidazole dose regimen in chronic chagasic patients: a systematic review}

\author{
Virgínia Paula Frade ${ }^{(D)}$, Nathália Soares Simões ${ }^{(1)}$, Nayara Ragi Baldoni ${ }^{\left({ }^{1}\right.}$, \\ Cristina Sanches ${ }^{\circledR 1}$, Cláudia Di Lorenzo Oliveira ${ }^{\circledR 1}$
}

\section{ABSTRACT}

The present study aimed to review the existing literature and to evaluate the best dose regimen for benznidazole in adult patients with Chagas disease in the chronic phase. A systematic review was conducted followed by meta-analysis. Searches were performed in four databases, to include studies published until May 2019. The descriptors used were: "Chagas disease", "benznidazole", "Drug Therapy", "Pharmacokinetics", "Dose-response relationship, drug" and "Chronic disease". The meta-analysis compared studies using the standard dose of $5 \mathrm{mg} / \mathrm{kg} /$ day for 30 or 60 days. A total of 608 articles were found, 23 of which were considered eligible for this review and nine were included in the meta-analysis. The studies selected and analyzed were published between 1996 and 2018, with various benznidazole dose regimens, ranging from $2.5 \mathrm{mg} / \mathrm{kg} / \mathrm{day}$ to $10 \mathrm{mg} / \mathrm{kg} / \mathrm{day}$, for 30 to 80 days of treatment. The results pointed to a great diversity of dose regimens, thus there is no consensus on the optimal dose regimen for benznidazole in the chronic phase of Chagas disease.

KEYWORDS: Benznidazole. Chagas disease. Drug therapy. Chronic phase.

\section{INTRODUCTION}

Chagas disease is classified as a neglected tropical disease caused by the protozoan Trypanosoma cruzi. It occurs in several countries, especially in Latin America, where it is estimated that about 6 to 8 million people are infected ${ }^{1}$. The disease is divided into two distinct phases: an initial acute phase and a chronic phase; the manifestations of the latter can take decades to occur ${ }^{2}$. The drugs available for its treatment are benznidazole and nifurtimox, which have limited efficacy and serious adverse effects, especially in the chronic phase ${ }^{1,3}$. Currently, benznidazole is considered the drug of choice for the treatment of the disease in Brazil $^{2,3}$.

The recommended dose of benznidazole for adult patients with Chagas disease in the chronic phase is $5 \mathrm{mg} / \mathrm{kg} /$ day, divided into two daily doses for a period of 60 days $^{4-6}$. However, adverse effects have been observed since the beginning of its use so that in 12 to $18 \%$ of adult patients at this stage of the disease, treatment has to be discontinued due to such effects. Thus, both, dose and duration of treatment are still being discussed in order to ensure greater patient safety ${ }^{6}$.

Diverging results in studies with the recommended dose have been found, especially regarding the duration of treatment. In the study conducted by Álvarez et al. ${ }^{6}$ no differences in the effectiveness of standard doses of benznidazole were observed between 30 and 60 consecutive days of administration, monitored by serial xenodiagnostics. In the study by Soy et al. $^{4}$, it was shown that the standard dose 
may be a significant overdose for some patients. According to Álvarez et al. ${ }^{6}$, the effective dose may be lower than the standard one proposed, and the best administration regimen to prevent or reduce adverse effects may not yet be known.

In addition, differences in the outcomes found in the studies could create barriers to treatment and even lead patients to experience worse health outcomes ${ }^{7}$. Thus, investigations are underway, and aiming at improving the therapy with benznidazole, its efficacy and safety, lower drug levels and shorter and/or intermittent regimens of the drug are being evaluated, either alone or in combination with other compounds ${ }^{8}$.

Given the above, the present study aimed to review the literature and evaluate the best dose regimen for benznidazole treatment in adult patients with Chagas disease in the chronic phase.

\section{MATERIALS AND METHODS}

\section{Study design}

This is a review followed by meta-analysis, performed according to PRISMA (Preferred Reporting Items for Systematic reviews and Meta-Analyses) ${ }^{9}$ guidelines.

\section{Elegibility criteria}

The "PICOS" process was established as: $\mathrm{P}$ (problem): adult patients with Chagas disease in the chronic phase; I (intervention): use of benznidazole; $\mathrm{C}$ (control): dose recommended by the literature; $\mathrm{O}$ (outcome): polymerase chain reaction (PCR) values, maintenance of cardiac conditions, negative seroconversion, reduction of clinical adverse events; S (study design): clinical trials and observational studies. According to "PICOS" the guiding question was defined: "What is the optimal dose regimen for benznidazole in adult patients with chronic Chagas disease?"

According to our inclusion criteria all the clinical trials or observational studies that evaluated adults patients with Chagas disease in the chronic phase and using benznidazole were considered eligible. Review articles, letters to the editor, opinion articles, studies on patients with Chagas disease using benznidazole only in the acute phase, preclinical studies, studies on pregnant women, children, and immunosuppressed patients were excluded.

\section{Information source}

The systematic review was performed in the PubMed, Science Direct, Virtual Health Library, and Scopus databases, including studies published until May 2019, with no restrictions on collection start date and language.

\section{Search strategy}

The descriptors were selected in consultation with the Medical Subject Heading (MeSH) and the following fixed descriptors were considered: "Chagas disease" and "benznidazole", which were cross referenced using the AND connector with: "Drug Therapy", "Pharmacokinetics", "Dose-response relationship, drug" and finally, with "Chronic disease". The selection, inclusion, and exclusion processes were performed by independent evaluators (VPF and NSS) and a third reviewer (CS), in case of disagreements.

\section{Methodological quality assessment}

Methodological quality was assessed by two reviewers (VPF and NSS), using the Downs and Black ${ }^{10}$ scores. This tool has 27 items that can be evaluated in five subclasses: communication, external validity, internal validity, selection bias and study power, with scores higher than 15 being considered as quality studies ${ }^{10}$.

\section{Data extraction}

After reading in full, the following variables from the articles that met the inclusion criteria were collected: year of publication, country, sample size, dose regimen used, follow-up period after treatment, observed outcomes, and limitations presented by the authors.

\section{Data analysis}

A large number of articles in which benznidazole was administered at $5 \mathrm{mg} / \mathrm{kg} /$ day during 30 or 60 days of treatment were found and they were all selected for analysis. The meta-analysis was calculated using a randomized $95 \%$ confidence interval model. The RStudio ${ }^{\circledR}$ software was used for this analysis. The meta and metafor packages were used, along with the metaprop and metabin commands. Statistical heterogeneity between studies was analyzed and inconsistency was estimated by Square $\left(\mathrm{I}^{2}\right)$ statistics, and considered up to $50 \%$. To explore this heterogeneity, timeseparated subgroup analyzes of benznidazole treatment were performed. The comparison between 30 and 60 days of treatment was made in order to evaluate the optimal treatment time of benznidazole at a dose of $5 \mathrm{mg} / \mathrm{kg} / \mathrm{day}$, observed through the outcomes: PCR, cardiac alterations and serology. In the articles that evaluated more than one 
outcome, the results were considered in the order in which they are cited above. We have also compared studies that used the PCR assay and/or negative seroconversion as an outcome.

\section{RESULTS}

A total of 608 articles were found and among them, 580 were selected for analysis as 28 were duplicates. After reading titles and abstracts, 543 studies were excluded because they were review articles, opinions and/or editorial letters, or articles with patients in the acute phase of Chagas disease, according to the exclusion criteria. A total of 37 studies were then selected for full reading. Of these, two did not meet our inclusion criteria because they included children, adolescents and/or immunosuppressed patients, and other twelve did not fit the inclusion criteria and were excluded. Therefore, 23 articles were included in the final analysis (Figure 1).

Of the included studies, $43.5 \%(\mathrm{n}=10)$ had a sample size smaller than or equal to 50 individuals $\mathrm{s}^{6,11-19}$ and $56.5 \%$ $(\mathrm{n}=13)$ greater than 50 individuals $^{20-32}$. Five articles
$(21.7 \%)^{11-13,19,21}$ had a higher risk of methodological bias, with scores lower than 15 in the quality assessment according to Downs and Black ${ }^{10}$, who claimed that the factors related to the potential confounders are the most affected.

The dose schedules of benznidazole used in the studies were diverse, ranging from $2.5 \mathrm{mg} / \mathrm{kg} / \mathrm{day}$ to $10 \mathrm{mg} / \mathrm{kg} / \mathrm{day}$, lasting 30 to 80 days of treatment. The predominant regimen was $5 \mathrm{mg} / \mathrm{kg} /$ day for 60 days, $39.1 \%(\mathrm{n}=9)^{6,12,13,16-19,28-30}$, followed by $5 \mathrm{mg} / \mathrm{kg} /$ day for 30 days, $17.4 \%(\mathrm{n}=4)^{22,24,31,32}$. Follow-up after benznidazole treatment ranged from 45 days to 21 years (Table 1 ).

Regarding the outcomes observed by the authors, $56.5 \%(\mathrm{n}=13)$ of the studies used the PCR assay $6,11,14,16,19-21,25-27,29,30,33$, and $43.5 \%(\mathrm{n}=10)$ evaluated the cardiac conditions of the participants ${ }^{12-14,16,20,22,23,26,31,32}$. A total of $43.5 \%(\mathrm{n}=10)$ used serological parameters $^{12-14,17,22-24,30-32}$ and $17.4 \%(n=4)$ used parasitological parameters to evaluate the benznidazole treatment ${ }^{14,15,22,23}$ (Table 1).

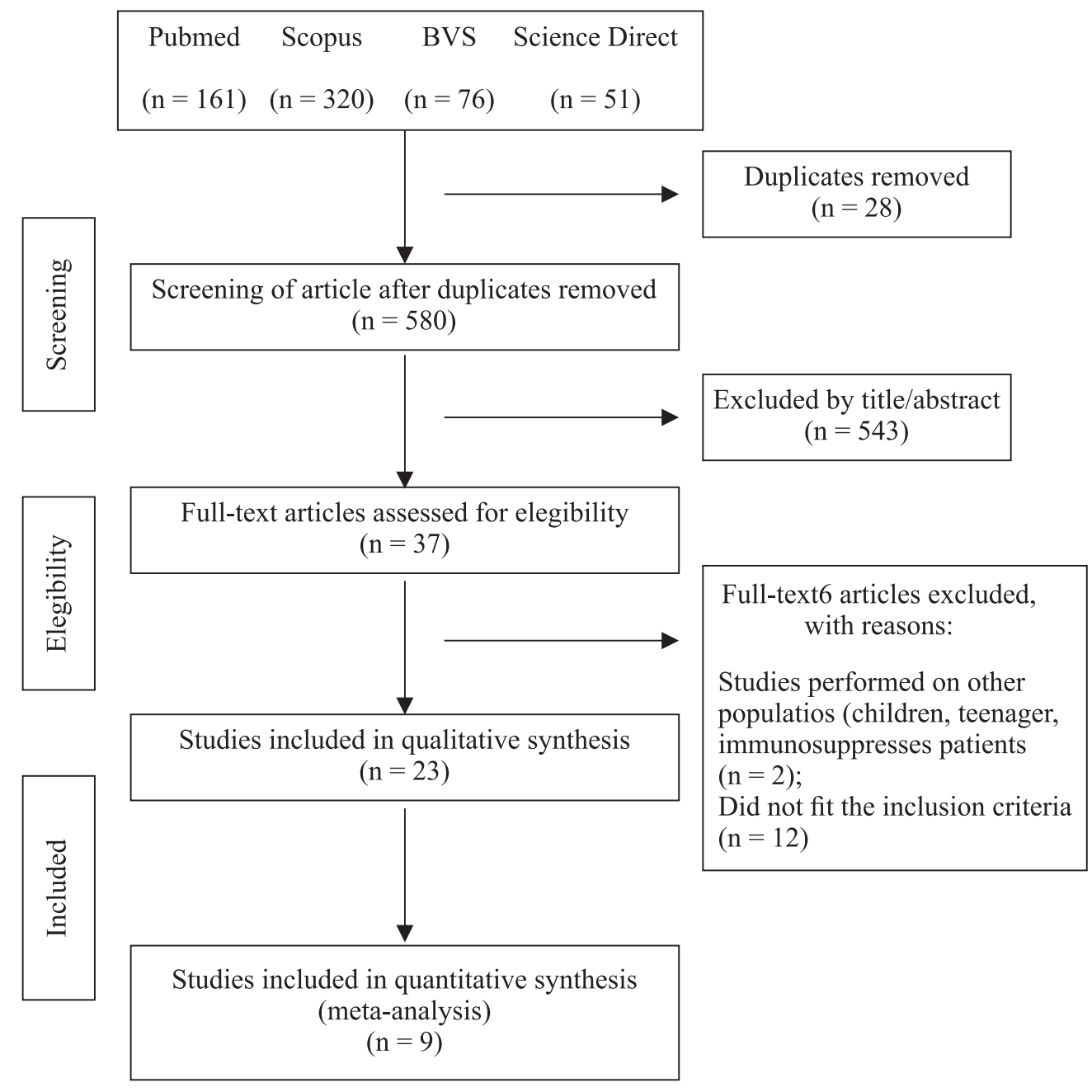

Figure 1 - Selection of items for the systematic review adapted from the PRISMA flowchart ${ }^{11}$. 
Table 1 - Studies evaluating benznidazole dosing regimens in adult patients with chronic Chagas disease $(n=23)$

\begin{tabular}{|c|c|c|c|c|c|}
\hline Author/Country & Sample size & $\begin{array}{l}\text { Treatment regimen of } \\
\text { benznidazole }\end{array}$ & $\begin{array}{l}\text { Follow-up period post } \\
\text { treatment }\end{array}$ & Analyzed outcome & Limitations \\
\hline $\begin{array}{l}\text { Alvarez et al. }{ }^{6} \\
\text { Argentina }\end{array}$ & $\begin{array}{l}\text { Total sample size } n=20 \\
\text { qPCR } n=17\end{array}$ & $\begin{array}{l}5 \mathrm{mg} / \mathrm{kg} / \mathrm{day} \text {, divided } \\
\text { into two daily doses } \\
\text { every } 5 \text { days, for a total } \\
\text { of } 60 \text { days ( } 12 \text { days of } \\
\text { intermittent treatment). }\end{array}$ & 67 days & $\begin{array}{c}\text { PCR } \\
\text { Adverse effects: } \\
\text { severity and } \\
\text { suspension rate }\end{array}$ & $\begin{array}{l}\text { The efficacy of } \\
\text { treatment as measured } \\
\text { by qPCR was limited to } \\
\text { a short-term follow-up } \\
\text { period. Thecomparison } \\
\text { of quantitative PCR } \\
\text { (qPCR) occurred before } \\
\text { and one week after the } \\
\text { end of treatment. . }\end{array}$ \\
\hline $\begin{array}{l}\text { Andrade et al. }{ }^{12} \\
\text { Brazil }\end{array}$ & $\begin{array}{l}\text { Total sample size } n=13 \\
\text { BNZ } n=13\end{array}$ & $5 \mathrm{mg} / \mathrm{kg} / \mathrm{day} / 60$ days & 4 years & $\begin{array}{c}\text { Serological parameters } \\
\text { Cardiac conditions }\end{array}$ & - \\
\hline $\begin{array}{l}\text { Andrade et al. }{ }^{13} \\
\text { Brazil }\end{array}$ & $\begin{array}{l}\text { Total sample size } n=30 \\
\text { BNZ } n=30\end{array}$ & $\begin{array}{c}5 \mathrm{mg} / \mathrm{kg} \text { every } 8 \text { hours/ } \\
60 \text { days }\end{array}$ & 6 years & $\begin{array}{l}\text { Serological } \\
\text { parameters } \\
\text { Cardiac } \\
\text { conditions }\end{array}$ & $\begin{array}{l}\text { Difficulty to perform } \\
\text { the follow-up for a long } \\
\text { period of time as the } \\
\text { patient's lived in rural } \\
\text { areas. }\end{array}$ \\
\hline $\begin{array}{l}\text { Antunes et al. }{ }^{20} \\
\text { Brazil }\end{array}$ & 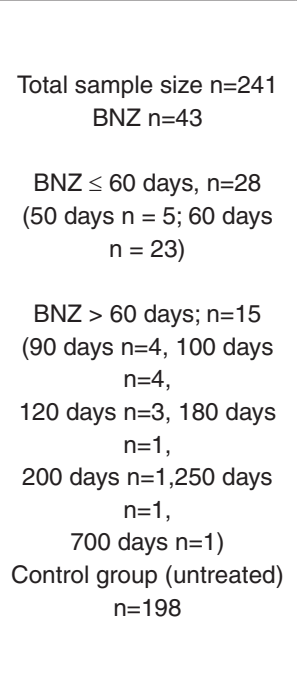 & $\begin{array}{l}\text { No dose information } \\
\text { BNZ for } 50-60 \text { days }\end{array}$ & Until 700 days & $\begin{array}{c}\text { PCR } \\
\text { Cardiac conditions }\end{array}$ & $\begin{array}{l}\text { Bias and the absence } \\
\text { of information in the } \\
\text { study population } \\
\text { relating to dosage and } \\
\text { the length of time after } \\
\text { treatment completion; } \\
\text { The adoption of } \\
\text { positive ELISA results } \\
\text { in the inclusion } \\
\text { criteria may have led } \\
\text { to the exclusion of } \\
\text { serologically cured } \\
\text { patients, thereby } \\
\text { reducing the ability } \\
\text { to detect differences } \\
\text { favoring the treatment; } \\
\text { The small sample } \\
\text { population probably } \\
\text { reduced the statistical } \\
\text { power to detect } \\
\text { differences. }\end{array}$ \\
\hline
\end{tabular}

\begin{tabular}{|c|c|c|c|c|c|}
\hline $\begin{array}{l}\text { Braga et al. }{ }^{21} \\
\text { Brazil }\end{array}$ & $\begin{array}{c}\text { Total sample size } n=51 \\
\text { BNZ } n=17 \\
\text { BNZ/30 days: } n=7 \\
\text { BNZ/60 days: } n=10 \\
\text { Untreated group } n=17 \\
\text { Control group (non-Chagas } \\
\text { individuals) } n=17\end{array}$ & $\begin{array}{c}10 \mathrm{mg} / \mathrm{Kg} / \text { day } / 30 \text { or } \\
60 \text { days }\end{array}$ & 10 years & $\begin{array}{c}\text { PCR } \\
\text { Molecular method }\end{array}$ & - \\
\hline $\begin{array}{l}\text { Coura et al. }{ }^{22} \\
\text { Brazil }\end{array}$ & $\begin{array}{c}\text { Total sample size } n=77 \\
\text { Nifurtimox } n=27 \\
\text { BNZ } n=26 \\
\text { Control group (placebo) } \\
n=24\end{array}$ & $\begin{array}{c}5 \mathrm{mg} / \mathrm{kg} / \mathrm{twice} \text { daily/ } \\
30 \text { days }\end{array}$ & 1 year & $\begin{array}{l}\text { Parasitological, } \\
\text { serological and clinical } \\
\text { parameters (cardiac } \\
\text { conditions) }\end{array}$ & - \\
\hline $\begin{array}{l}\text { Fabbro et al. }{ }^{23} \\
\text { Argentina }\end{array}$ & $\begin{array}{l}\text { Total sample size } n=111 \\
\text { Nirfutimox } n=27 \\
\text { BNZ } n=27 \\
\text { Control group (untreated) } \\
\quad n=57\end{array}$ & $\begin{array}{l}5 \mathrm{mg} / \mathrm{kg} / \mathrm{day} / 30 \text { days } \\
\text { with half of the dose in } \\
\text { the first week. }\end{array}$ & 21 years & $\begin{array}{l}\text { Parasitological, } \\
\text { serological and } \\
\text { clinical parameters } \\
\text { (cardiac conditions) }\end{array}$ & $\begin{array}{l}\text { There was lack of } \\
\text { randomization of } \\
\text { the groups, as most } \\
\text { infected patients whose } \\
\text { xenodiagnosis were } \\
\text { positive, received } \\
\text { treatment. }\end{array}$ \\
\hline $\begin{array}{l}\text { Fernández et al. }{ }^{11} \\
\text { Argentina }\end{array}$ & $\begin{array}{l}\text { Total sample size } n=6 \\
\text { BNZ } n=6\end{array}$ & $\begin{array}{c}2.5-5.5 \mathrm{mg} / \mathrm{Kg} / 60 \text { days } \\
(2.50,2.60,4.12,4.55 \\
4.55,5.48)\end{array}$ & 06 months & PCR & - \\
\hline $\begin{array}{l}\text { Lana et al. }{ }^{14} \\
\text { Brazil }\end{array}$ & $\begin{array}{l}\text { Total sample size } n=28 \\
\text { BNZ } n=28\end{array}$ & $\begin{array}{c}5-10 \mathrm{mg} / \mathrm{kg} / \text { day for } \\
40-60 \text { consecutive days }\end{array}$ & 9 years & $\begin{array}{c}\text { PCR } \\
\text { Parasitological, } \\
\text { serological and clinical } \\
\text { parameters (cardiac } \\
\text { conditions) }\end{array}$ & $\begin{array}{c}\text { There was a low } \\
\text { number of available } \\
\text { samples. } \\
\text { There was no intrinsic } \\
\text { control group. }\end{array}$ \\
\hline
\end{tabular}


Table 1 - Studies evaluating benznidazole dosing regimens in adult patients with chronic Chagas disease $(n=23)$

\begin{tabular}{|c|c|c|c|c|}
\hline Author/Country & Sample size & $\begin{array}{l}\text { Treatment regimen of } \\
\text { benznidazole }\end{array}$ & $\begin{array}{c}\text { Follow-up period post } \\
\text { treatment }\end{array}$ & Analyzed outcome \\
\hline $\begin{array}{l}\text { Laucella et al. }{ }^{24} \\
\text { Argentina }\end{array}$ & $\begin{array}{l}\text { Total sample size } n=75 \\
\text { BNZ } n=43 \\
\text { Control group (untreated) } \\
n=32\end{array}$ & $\begin{array}{c}5 \mathrm{mg} / \mathrm{kg} / \mathrm{day} / \\
30 \text { days }\end{array}$ & $3-5$ years & $\begin{array}{l}\text { Immunological, } \\
\text { serological and clinical } \\
\text { parameters }\end{array}$ \\
\hline $\begin{array}{l}\text { Levi et al. }{ }^{15} \\
\text { Brazil }\end{array}$ & $\begin{array}{c}\text { Total sample size } n=49 \\
\text { BNZ } n=41 \\
\text { Nirfutlmox } n=8\end{array}$ & $\begin{array}{c}5 \mathrm{mg} / \mathrm{kg} / \mathrm{day} \text { to } \\
8 \mathrm{mg} / \mathrm{kg} / \mathrm{day} / 60 \text { days }\end{array}$ & $\begin{array}{c}\text { Ranged from } 1 \text { to } 20 \\
\text { years (mean: } 6 \text { years. } 7 \\
\text { months). }\end{array}$ & $\begin{array}{l}\text { Parasitological } \\
\text { parameters }\end{array}$ \\
\hline $\begin{array}{l}\text { Molina et al. }{ }^{25} \\
\text { Bolivia, Brazil and } \\
\text { Paraguay }\end{array}$ & $\begin{array}{l}\text { Total sample size } n=78 \\
\qquad \text { BNZ } n=26 \\
\text { High-dose POS } n=26 \\
\text { Low-dose POS } n=26\end{array}$ & $\begin{array}{c}150 \mathrm{mg} \text { twice daily/ } \\
60 \text { days }\end{array}$ & 10 months & $\begin{array}{l}\text { PCR } \\
\text { Adverse effects: } \\
\text { suspension rate }\end{array}$ \\
\hline $\begin{array}{l}\text { Morillo et al. }{ }^{26} \\
\text { Argentina, Bolivia, } \\
\text { Brazil, Colombia and El } \\
\text { Salvador }\end{array}$ & $\begin{array}{l}\text { Total sample size } n=2854 \\
\qquad \text { BNZ } n=1431 \\
\text { Control group } \\
\text { (placebo) } n=1423\end{array}$ & $\begin{array}{l}300 \text { mg per day with } \\
\text { a variable duration of } \\
\text { therapy ( } 40 \text { to } 80 \text { days) }\end{array}$ & 5.4 years & $\begin{array}{c}\text { PCR } \\
\text { Cardiac conditions }\end{array}$ \\
\hline $\begin{array}{l}\text { Morillo et al. }{ }^{27} \\
\text { Argentina, Chile, } \\
\text { Colombia, Guatemala, } \\
\text { Mexico and Spain }\end{array}$ & $\begin{array}{l}\text { Total sample size } n=120 \\
\text { POS } n=30 \\
\text { BNZ+placebo } n=30 \\
\text { POS }+ \text { BNZ } n=30 \\
\text { Control group } \\
\text { (placebo) } n=30\end{array}$ & $\begin{array}{c}\text { POS: } 400 \mathrm{mg} \text { twice } \\
\text { daily/60 days } \\
\text { BNZ+placebo: } 200 \mathrm{mg} \\
\text { daily/60 days } \\
\text { POS+BNZ: } 400+200 \mathrm{mg} \\
\text { twice daily/60 days } \\
\text { Placebo: } 10 \mathrm{mg} \text { twice } \\
\text { daily/ } 60 \text { days }\end{array}$ & 1 year & PCR \\
\hline
\end{tabular}

PCR results were not used as a measure of fficacy or cure, only as a marker of treatment failure.

The 95\% confidence intervals rule out a relative risk reduction of $20 \%$, which is smaller than the difference that the study was designed to detect.

Follow-up was limited to only one year and the effect of trypanocidal treatment on progression to cardiomyopathy is unknown.

The small number of treated patients.

Most subjects in the chronic phase of the disease did not fulfill

Oliveira et al. ${ }^{16}$ Brazil
Total sample size $n=45$

$$
\text { BNZ } n=21
$$

Control group (untreated) $n=24$
$5 \mathrm{mg} / \mathrm{kg}$ twice daily/60 days
6 months

6 months
Blood culture

PCR

Cardiac conditions the inclusion criteria, limiting the number of patients

The effects of BNZ during treatment or for a long time after treatment were not investigated.

\begin{tabular}{|c|c|c|c|c|c|}
\hline & & & & & \\
\hline $\begin{array}{l}\text { Pérez-Antón et al. }{ }^{19} \\
\text { Spain }\end{array}$ & $\begin{array}{c}\text { Total sample size } n=50 \\
\text { BNZ } n=38 \\
\text { Control group (non-Chagas } \\
\text { individuals) } n=12\end{array}$ & $5 \mathrm{mg} / \mathrm{kg} / \mathrm{day} / 60$ days & \multicolumn{2}{|r|}{$\begin{array}{c}\text { PCR } \\
\text { Immunological } \\
\text { parameters }\end{array}$} & - \\
\hline $\begin{array}{l}\text { Perez-Mazliah et al. }{ }^{17} \\
\text { Argentina }\end{array}$ & $\begin{array}{c}\text { Total sample size } n=50 \\
\text { Allopurinol and BNZ } n=15 \\
\text { Untreated subjects } n=17 \\
\text { BNZ alone } n=9 \\
\text { Control group (non-Chagas } \\
\text { individuals) } n=9\end{array}$ & $\begin{array}{c}\text { Allopurinol: } \\
600 \mathrm{mg} / \text { day } / 90 \text { days } \\
\text { followed by } 30 \text { days of } \\
\text { BNZ ( } 5 \mathrm{mg} / \mathrm{kg} / \text { day })\end{array}$ & 3 years & $\begin{array}{l}\text { Immunological, } \\
\text { Serological and clinical } \\
\text { parameters }\end{array}$ & - \\
\hline $\begin{array}{l}\text { Pinazo et al. }{ }^{28} \\
\text { Spain }\end{array}$ & $\begin{array}{l}\text { Total sample size } n=54 \\
\text { BNZ } n=54\end{array}$ & $\begin{array}{c}5 \mathrm{mg} / \mathrm{kg} / \mathrm{day} / \\
60 \text { days }\end{array}$ & 1 year & $\begin{array}{c}\text { Adverse effects } \\
\text { Biochemical and } \\
\text { hematology testing }\end{array}$ & $\begin{array}{l}\text { The serum } \\
\text { concentrations } \\
\text { of benznidazole } \\
\text { metabolites were not } \\
\text { measured and they } \\
\text { might play an important } \\
\text { role in toxicity. }\end{array}$ \\
\hline
\end{tabular}


Table 1 - Studies evaluating benznidazole dosing regimens in adult patients with chronic Chagas disease $(n=23)$

\begin{tabular}{|c|c|c|c|c|c|}
\hline Author/Country & Sample size & $\begin{array}{c}\text { Treatment regimen of } \\
\text { benznidazole }\end{array}$ & $\begin{array}{c}\text { Follow-up period post } \\
\text { treatment }\end{array}$ & Analyzed outcome & Limitations \\
\hline $\begin{array}{l}\text { Pinazo et al. }{ }^{29} \\
\text { Spain }\end{array}$ & $\begin{array}{c}\text { Total sample size } n=99 \\
\text { BNZ } n=56 \\
\text { Control group (non-Chagas } \\
\text { individuals) } n=43\end{array}$ & $\begin{array}{l}5 \mathrm{mg} / \mathrm{kg} / \text { day } / \\
60 \text { days }\end{array}$ & 3 years & $\begin{array}{l}\text { Hemostasis tests } \\
\text { PCR } \\
\text { Biochemical testing } \\
\text { Basic blood testing }\end{array}$ & $\begin{array}{c}\text { A larger sample } \\
\text { could have detected } \\
\text { differences that would } \\
\text { be expected to appear } \\
\text { earlier (e.g., before } 12 \\
\text { months). } \\
\text { The lost to follow-up } \\
\text { samples affected the } \\
\text { estimates. } \\
\text { It is difficult to follow-up } \\
\text { individuals with high } \\
\text { migratory mobility for } \\
\text { long periods. } \\
\text { The fact that only } \\
\text { 30\% of patients had } \\
\text { a positive baseline } \\
\text { qRT-PCR result was a } \\
\text { constraint for assessing } \\
\text { the effect of treatment. }\end{array}$ \\
\hline $\begin{array}{l}\text { Torrico et al. }{ }^{30} \\
\text { Bolivia }\end{array}$ & $\begin{array}{l}\text { Total sample size } n=560 \\
\text { BNZ } n=45 \\
\text { Control group (untreated) } \\
n=89\end{array}$ & $\begin{array}{l}5 \mathrm{mg} / \mathrm{kg} / \text { day } / \\
60 \text { days }\end{array}$ & 1 year & $\begin{array}{c}\text { PCR } \\
\text { Serological parameters }\end{array}$ & - \\
\hline $\begin{array}{l}\text { Vallejo et al. }{ }^{33} \\
\text { Spain }\end{array}$ & $\begin{array}{l}\text { Total sample size } n=14 \\
\text { BNZ } n=7 \\
\text { Control group (untreated) } \\
\qquad n=7\end{array}$ & $\begin{array}{c}5 \mathrm{mg} / \mathrm{kg} \text { twice daily/ } \\
60 \text { days }\end{array}$ & 18 months & $\begin{array}{c}\text { PCR } \\
\text { Immune parameters }\end{array}$ & $\begin{array}{l}\text { Small sample size. } \\
\text { Problems with the } \\
\text { follow-up of some } \\
\text { patients that have } \\
\text { impaired the analysis of } \\
\text { parameters at specific } \\
\text { time points. }\end{array}$ \\
\hline $\begin{array}{l}\text { Viotti et al. }{ }^{31} \\
\text { Argentina }\end{array}$ & $\begin{array}{c}\text { Total sample size } n=566 \\
\text { BNZ } n=283 \\
\text { Control group (untreated) } \\
n=283\end{array}$ & $\begin{array}{l}5 \mathrm{mg} / \mathrm{kg} / \mathrm{day} / \\
30 \text { days }\end{array}$ & Median time 9.8 years & $\begin{array}{l}\text { Serological parameters } \\
\text { Cardiac conditions } \\
\text { Disease progression } \\
\text { (defined as a change to a } \\
\text { more advanced Kuschnir } \\
\text { group or death). }\end{array}$ & $\begin{array}{c}\text { Non-random, unblinded } \\
\text { treatment assignment } \\
\text { was used, and follow-up } \\
\text { data were missing for } \\
20 \% \text { of the patients. } \\
\text { Loss to follow-up was } \\
\text { more common among } \\
\text { patients who were less } \\
\text { sick. }\end{array}$ \\
\hline $\begin{array}{l}\text { Viotti et al. }{ }^{32} \\
\text { Argentina }\end{array}$ & $\begin{array}{l}\text { Total sample size } n=142 \\
\text { BNZ } n=53 \\
\text { Control group (untreated) } \\
n=89\end{array}$ & $\begin{array}{l}5 \mathrm{mg} / \mathrm{kg} / \mathrm{day} / \\
30 \text { days }\end{array}$ & 3 years & $\begin{array}{l}\text { Serological parameters } \\
\text { Cardiac conditions }\end{array}$ & $\begin{array}{l}\text { The non-randomized } \\
\text { design. } \\
\text { Results should be } \\
\text { also extrapolated with } \\
\text { caution due to the } \\
\text { possibility that diverse } \\
\text { T. cruzi lineages } \\
\text { may have different } \\
\text { susceptibilities to BNZ. }\end{array}$ \\
\hline
\end{tabular}

$\mathrm{POS}=$ Posaconazole; $\mathrm{qPCR}=$ quantitative polymerase chain reaction; F1+2 $=$ Prothrombin fragment $1+2 ; \mathrm{ETP}=$ endogenous thrombin potential; PAP $=$ plasmin-antiplasmin complexes

The main limitations observed in the studies were difficulties during the follow-up period, such as loss or short follow-up time, $30.4 \%(\mathrm{n}=7)^{6,13,16,18,27,29,31}$, in addition to the small sample size, $21.7 \%(\mathrm{n}=5)^{14,16,18,20,29}$ (Table 1).

Negative qualitative PCR results were found in all the studies that used PCR as an outcome, and 14.8 to $100.0 \%$ of negative patients after treatment were observed $^{6,11,14,16,18-20,25-27,29,30}$. Cases of cardiac conditions worsening were found in $60.0 \%(\mathrm{n}=8)$ of the studies that evaluated these conditions, with 3.7 to $38.2 \%$ of participants with such worsening conditions ${ }^{12,13,17,23,24,30-32}$. Finally, cases of seronegativation were observed in $80 \%(n=8)$ of the studies evaluating this parameter, being observed from 4.6 to $73.0 \%$ of participants with negative serology after benznidazole use ${ }^{12,13,17,23,24,30-32}$. In $60.9 \%(n=14)$ of the studies, adverse effects were reported, such as: gastrointestinal symptoms, dermatitis, cutaneous reactions, among others $6,11,14,15,17,18,20,22,23,25-28,30$ (Table 2). 
Table 2 - Negative PCR results, cardiac conditions, seronegativation; negative parasitological tests after treatment with benznidazole and adverse events observed during the treatment.

\begin{tabular}{|c|c|c|c|c|c|c|}
\hline Author & $\begin{array}{l}\text { Treatment scheme of } \\
\text { benznidazole }\end{array}$ & PCR results (\%) & $\begin{array}{c}\text { Cardiac conditions } \\
(\%)\end{array}$ & $\begin{array}{c}\text { Serological } \\
\text { parameters (\%) }\end{array}$ & $\begin{array}{c}\text { Parasitological } \\
\text { parameters } \\
\text { negative results } \\
(\%)\end{array}$ & Adverse effects \\
\hline Alvarez et al. ${ }^{6}$ & $\begin{array}{l}5 \mathrm{mg} / \mathrm{kg} / \text { day, divided } \\
\text { into two daily doses } \\
\text { every } 5 \text { days, for a } \\
\text { total of } 60 \text { days }\end{array}$ & 94.0 & - & - & - & $\begin{array}{c}\text { Dermatitis (35.0\%); } \\
\text { gastrointestinal intolerance } \\
(25.0 \%) \text {; increased liver } \\
\text { enzymes }(20.0 \%) \text {; loss of } \\
\text { appetite }(5.0 \%) \text {; insomnia } \\
(5.0 \%) \text {. No serious } \\
\text { adverse effects. }\end{array}$ \\
\hline Andrade et al. ${ }^{12}$ & $5 \mathrm{mg} / \mathrm{kg} / \mathrm{day} / 60$ days & - & Maintained: 62.9 & $\begin{array}{l}\text { Unchanged: } 69.2 \\
\text { Regressive: } 23.1 \\
\text { Progressive: } 7.7\end{array}$ & - & - \\
\hline Andrade et al..$^{13}$ & $\begin{array}{c}5 \text { mg/kg every } 8 \\
\text { hours/60 days }\end{array}$ & - & $\begin{array}{l}\text { Maintained: } 76.2 \\
\text { Worsening: } 19.0\end{array}$ & $\begin{array}{l}\text { Unchanged: } 80.0 \\
\text { Regressive: } 10.0 \\
\text { Progressive: } 10.0\end{array}$ & & - \\
\hline Antunes et al. ${ }^{20}$ & $\begin{array}{c}\text { No dose information. } \\
\text { BNZ for } 50-60 \text { days; } \\
\text { BNZ > } 60 \text { days. }\end{array}$ & $\begin{array}{c}\text { BNZ } \leq 60 \text { days: } 77.8 \\
\text { BNZ }>60: 66.7\end{array}$ & Maintained: 100.0 & - & - & Allergic reactions (19.5\%) \\
\hline Braga et al. ${ }^{21}$ & $\begin{array}{l}10 \mathrm{mg} / \mathrm{Kg} / \text { day/ } \\
30 \text { or } 60 \text { days }\end{array}$ & $\begin{array}{l}<1 \text { to } 36 \text { T. cruzi/ml } \\
\text { (means } 6.45 \pm 9.28 \text { ) }\end{array}$ & - & - & - & - \\
\hline Coura et al. ${ }^{22}$ & $\begin{array}{c}5 \mathrm{mg} / \mathrm{kg} / \mathrm{twice} \text { daily/ } \\
30 \text { days }\end{array}$ & - & Maintained: 100.0 & Unchanged: 100.0 & 98.2 & $\begin{array}{c}\text { Rash, peripheral } \\
\text { neuropathy } \\
\text { gastrointestinal disorders. } \\
\text { The adverse effects were } \\
\text { discrete or moderate. }\end{array}$ \\
\hline Fabbro et al. ${ }^{23}$ & $\begin{array}{l}5 \mathrm{mg} / \mathrm{kg} / \text { day/ } \\
30 \text { days with half of } \\
\text { the dose in the first } \\
\text { week. }\end{array}$ & - & Worsening: 3.7 & $\begin{array}{l}\text { Unchanged: } 44,4 \\
\text { Regressive: } 33,3 \\
\text { Progressive: } 22,2\end{array}$ & 100.0 & $\begin{array}{c}\text { Maculopapular erythema } \\
\text { (12.5\%); pruritus and } \\
\text { edema (3.7\%). These } \\
\text { patients had to stop the } \\
\text { medication because of } \\
\text { intolerance. }\end{array}$ \\
\hline Fernández et al. ${ }^{11}$ & $\begin{array}{c}2.5-5.5 \mathrm{mg} / \mathrm{Kg} / \\
60 \text { days }\end{array}$ & 100.0 & - & - & - & $\begin{array}{c}\text { Dermatitis, pruritus, oral } \\
\text { mucositis, dysgeusia, } \\
\text { paresthesia in lower limb, } \\
\text { hypereosinophilia. }\end{array}$ \\
\hline Lana et al. ${ }^{14}$ & $\begin{array}{c}5-10 \mathrm{mg} / \mathrm{kg} / \text { day for } \\
40-60 \text { consecutive } \\
\text { days }\end{array}$ & 14.8 & Worsening: 4.6 & Unchanged: 100.0 & 92.9 & $\begin{array}{c}\text { Anorexia, muscle pain, } \\
\text { exanthema. One case of } \\
\text { severe adverse effects } \\
\text { was documented. }\end{array}$ \\
\hline Laucella et al. ${ }^{24}$ & $5 \mathrm{mg} / \mathrm{kg} / \mathrm{day} / 30$ days & - & - & $\begin{array}{l}\text { Unchanged: } 81.5 \\
\text { Regressive: } 13.9 \\
\text { Progressive: } 4.6\end{array}$ & - & - \\
\hline Levi et al. ${ }^{15}$ & $\begin{array}{c}5 \mathrm{mg} / \mathrm{kg} / \mathrm{day} \text { to } 8 \mathrm{mg} / \\
\mathrm{kg} / \mathrm{day} / 60 \text { days }\end{array}$ & - & - & - & 63.4 & $\begin{array}{l}\text { Vesicular rash, peripheral } \\
\text { neuropathy and psychiatric } \\
\text { manifestations, leading } \\
\text { to discontinuation of } \\
\text { treatment in } 41.5 \% \text { of the } \\
\text { patients. }\end{array}$ \\
\hline Molina et al..$^{25}$ & $\begin{array}{c}150 \text { mg twice daily/ } \\
60 \text { days }\end{array}$ & 61.5 & - & - & - & $\begin{array}{c}\text { Severe cutaneous } \\
\text { reactions }(6.4 \%) \text { leading } \\
\text { to discontinuation of } \\
\text { treatment. }\end{array}$ \\
\hline Morillo et al. ${ }^{26}$ & $\begin{array}{l}300 \text { mg per day with } \\
\text { a variable duration of } \\
\text { therapy (between } \\
40 \text { to } 80 \text { days) }\end{array}$ & $\begin{array}{l}\text { At the end of the } \\
\text { treatment: } 66.2 ; \\
2 \text { years: } 55.4 ; \\
5 \text { years } \\
\text { or more: } 46.7\end{array}$ & $\begin{array}{c}\text { Worsening at } 2 \text { years: } \\
36.7 \text {; Worsening at } \\
5 \text { years or more: } 38.2\end{array}$ & - & - & $\begin{array}{c}\text { Cutaneous rash, } \\
\text { gastrointestinal symptoms, } \\
\text { and nervous system } \\
\text { disorders leading to } \\
\text { discontinuation of } \\
\text { treatment in } 23.9 \% \text { of the } \\
\text { patients. }\end{array}$ \\
\hline
\end{tabular}


Table 2 - Negative PCR results, cardiac conditions, seronegativation; negative parasitological tests after treatment with benznidazole and adverse events observed during the treatment.

\begin{tabular}{|c|c|c|c|c|c|c|}
\hline Author & $\begin{array}{c}\text { Treatment scheme of } \\
\text { benznidazole }\end{array}$ & PCR results (\%) & $\begin{array}{c}\text { Cardiac conditions } \\
(\%)\end{array}$ & $\begin{array}{c}\text { Serological } \\
\text { parameters (\%) }\end{array}$ & $\begin{array}{c}\text { Parasitological } \\
\text { parameters } \\
\text { negative results } \\
(\%)\end{array}$ & Adverse effects \\
\hline Morillo et al.. ${ }^{27}$ & $\begin{array}{l}20 \text { 0mg daily/ } \\
60 \text { days }\end{array}$ & $\begin{array}{c}\text { BNZ+POS: } 30 \text { days: } \\
88.960 \text {; days: } 92.3 ; \\
360 \text { days: } 96.0 \\
\text { BNZ: } 30 \text { days: } 89.7 ; \\
60 \text { days: } 89.3 ; \\
360 \text { days: } 96.0\end{array}$ & - & - & - & $\begin{array}{l}\text { Severe adverse events } \\
(7.0 \%) \text { : cutaneous rash, } \\
\text { nervous system disorders } \\
\text { and gastrointestinal signs } \\
\text { and symptoms. }\end{array}$ \\
\hline Oliveira et al. ${ }^{16}$ & $\begin{array}{c}5 \mathrm{mg} / \mathrm{kg} \text { twice daily/ } \\
60 \text { days }\end{array}$ & 76.2 & $\begin{array}{c}\text { There was worsening } \\
\text { of the heart rate. }\end{array}$ & - & - & - \\
\hline Pérez-Antón et al. ${ }^{19}$ & $\begin{array}{c}5 \mathrm{mg} / \mathrm{kg} / \text { day } / \\
60 \text { days }\end{array}$ & 52.6 & - & - & - & - \\
\hline Perez- Mazliah et al. ${ }^{17}$ & $\begin{array}{c}\text { Allopurinol: } \\
600 \mathrm{mg} / \text { day/ } \\
90 \text { days followed by } \\
30 \text { days of BNZ ( } 5 \\
\mathrm{mg} / \mathrm{kg} / \text { day) }\end{array}$ & - & - & Regressive: 73.0 & - & $\begin{array}{c}\text { Mild dermatitis }(26.7 \%) \text {, } \\
\text { moderate dermatitis } \\
(26.7 \%) \text {. Treatment } \\
\text { discontinuation was } \\
\text { required in two cases } \\
(13 \%) \text { : one during } \\
\text { treatment with allopurinol } \\
\text { and one during treatment } \\
\text { with BNZ. }\end{array}$ \\
\hline Pinazo et al..$^{28}$ & $\begin{array}{c}5 \mathrm{mg} / \mathrm{kg} / \mathrm{day} / \\
60 \text { days }\end{array}$ & - & - & - & 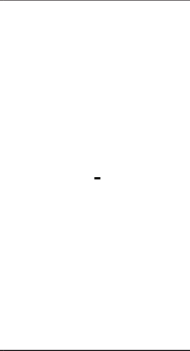 & $\begin{array}{c}\text { Gastrointestinal symptoms } \\
(76.0 \%) ; \text { skin symptoms } \\
(74.0 \%), \text { neurological/ } \\
\text { musculoskeletal symptoms } \\
(74.0 \%), \text { pruritus }(70.0), \\
\text { fever }(15.0 \%) \text {. Treatment } \\
\text { was discontinued in } 11 \\
\text { patients, } 7 \text { among them } \\
\text { due to severe adverse } \\
\text { effects. }\end{array}$ \\
\hline Pinazo et al..$^{29}$ & $\begin{array}{c}5 \mathrm{mg} / \mathrm{kg} / \text { day } / \\
60 \text { days }\end{array}$ & 75.0 & - & - & - & - \\
\hline Torrico et al..$^{30}$ & $\begin{array}{c}5 \mathrm{mg} / \mathrm{kg} \text { twice daily/ } \\
60 \text { days }\end{array}$ & 97.0 & - & $\begin{array}{c}\text { Unchanged: } 87.0 \\
\text { Regressive: } 9.0 \\
\text { Inconclusive: } 4.0\end{array}$ & - & $\begin{array}{c}\text { Severe adverse events: } \\
4.0 \%\end{array}$ \\
\hline Vallejo et al. ${ }^{33}$ & $\begin{array}{c}5 \mathrm{mg} / \mathrm{kg} \text { twice daily/ } \\
60 \text { days }\end{array}$ & 100.0 & - & - & - & $\begin{array}{l}\text { Maculopapular rash, } \\
\text { facial rash, fever, rash. } \\
\text { Three discontinued } \\
\text { therapy owing to adverse } \\
\text { reactions. }\end{array}$ \\
\hline Viotti et al. ${ }^{31}$ & $5 \mathrm{mg} / \mathrm{kg} /$ day $/ 30$ days & - & Worsening: 5.0 & Regressive: 15.0 & - & - \\
\hline Viotti et al. ${ }^{32}$ & $5 \mathrm{mg} / \mathrm{kg} /$ day $/ 30$ days & - & Maintained: 100.0 & Regressive: 40.0 & - & - \\
\hline
\end{tabular}

The clinical heterogeneity of the studies included in the meta-analysis was statistically significant, with an $\mathrm{I}^{2}$ of $90.0 \%$ for the nine articles ( $<<0.01)$ (Table 3). For the group that used benznidazole for 60 days, $\mathrm{I}^{2}$ was $84 \%$ and for the group that used for 30 days, $\mathrm{I}^{2}$ was $60 \%$. Pooled data from studies that used benznidazole for 30 days tended to have better results of the observed parameters (PCR, cardiac alterations and seroconversion) after treatment than those that used benznidazole for 60 days. For those who used PCR as the outcome, the risk of a negative result was 0.41 times higher after the end of treatment (Table 4) and for those who were analyzed by serology, the risk of negative serology was 3.65 times higher after treatment (Table 5).

\section{DISCUSSION}

The present review evaluated 23 studies that tested different benznidazole dose regimens in the chronic phase of Chagas disease. Our results pointed out that there was no consensus in the literature regarding dose, treatment time and cure criteria.

In most studies, the standard dose of $5 \mathrm{mg} / \mathrm{kg} / \mathrm{day}$ was 
Table 3 - Evolution of PCR parameters, cardiac conditions and serology among studies on benznidazole treatment for 30 and 60 days.

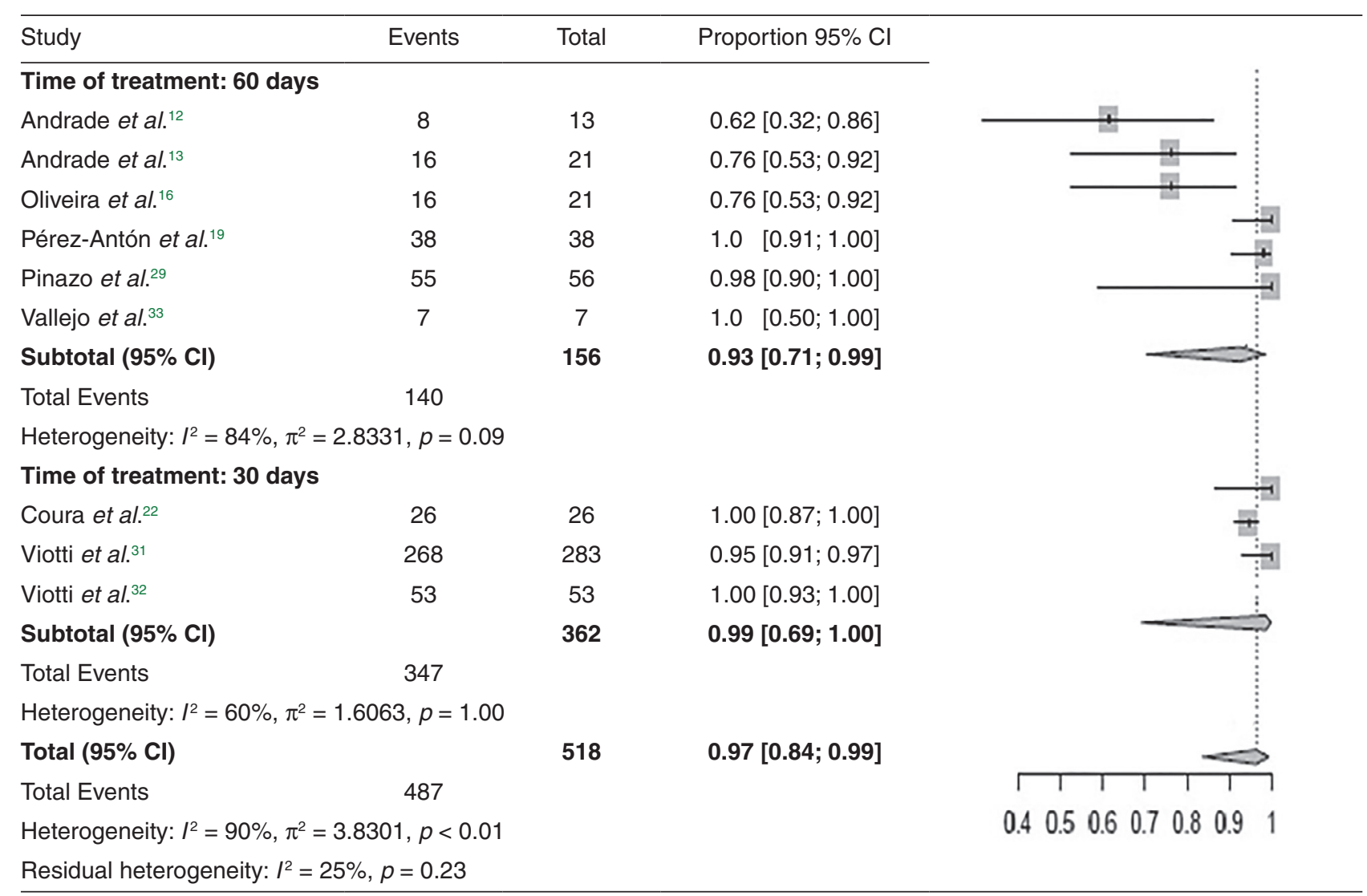

Table 4 - Risk of negative PCR results before and after benznidazole treatment.

\begin{tabular}{|c|c|c|c|c|c|c|c|c|c|c|c|}
\hline \multirow{2}{*}{ Study } & \multicolumn{2}{|c|}{ Before } & \multicolumn{2}{|c|}{ After } & \multirow{2}{*}{ Weight } & \multirow{2}{*}{$\begin{array}{c}\text { RR } \\
95 \% \mathrm{Cl}\end{array}$} & \multirow{3}{*}{\multicolumn{3}{|c|}{ 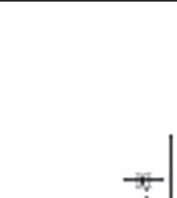 }} & & \\
\hline & Events & Total & Events & Total & & & & & & & \\
\hline Alvarez et al. ${ }^{6}$ & 6 & 17 & 16 & 17 & $25.4 \%$ & $0.38[0.19 ; 0.72]$ & & & & & \\
\hline Oliveira et al. ${ }^{16}$ & 0 & 21 & 16 & 21 & $4.8 \%$ & $0.03[0.00 ; 0.47]$ & \multirow{2}{*}{\multicolumn{3}{|c|}{ 邹 }} & & \\
\hline Pérez-Antón et al. ${ }^{19}$ & 18 & 38 & 38 & 38 & $31.4 \%$ & $0.48[0.35 ; 0.67]$ & & & & & \\
\hline Pinazo et al. ${ }^{29}$ & 40 & 56 & 55 & 56 & $41.2 \%$ & $0.73[0.61 ; 0.86]$ & & & \\
\hline Vallejo et al..$^{33}$ & 0 & 7 & 7 & 7 & $5.6 \%$ & $0.07[0.00 ; 0.97]$ & \multirow{2}{*}{\multicolumn{3}{|c|}{$\dot{\infty}$}} & & \\
\hline Total $(95 \% \mathrm{Cl})$ & & 139 & & 139 & & $0.41[0.21 ; 0.79]$ & & & & & \\
\hline Total events & \multicolumn{2}{|c|}{64} & 132 & & & & \multirow[t]{2}{*}{0.01} & 0.1 & 1 & $\begin{array}{c}1 \\
10\end{array}$ & ${ }_{100}$ \\
\hline \multicolumn{7}{|c|}{ Heterogeneity: $I^{2}=85 \%, \mathrm{t}^{2}=0.3255, p<0.01$} & & & & & \\
\hline
\end{tabular}

used, only varying the treatment time. Comparing the studies that used this dose for 30 and 60 days, it was observed that long-term treatment does not bring great benefits to the user. Through the meta-analysis, it was noticed that those who used benznidazole for a shorter time showed a tendency of better results of the observed parameters.

One great difficulty in the follow up of patients with Chagas disease is criteria to define cure, because there is not a consensual biomarker. The confirmation of cure varies according to the duration of disease, age, comorbidities, tests used, and time of follow-up after treatment ${ }^{34,35}$.

Thus, it must be taken into account that the outcomes evaluated in the studies included in the meta-analysis are complex and have a heterogeneous distribution. In addition, we analyzed cardiac conditions without observing the clinical form of the chronic disease at baseline, considering only the improvement or maintenance of initial conditions. In the groups of the included studies, the participants' 
Table 5 - Relative risk of negative serology after benznidazole treatment.

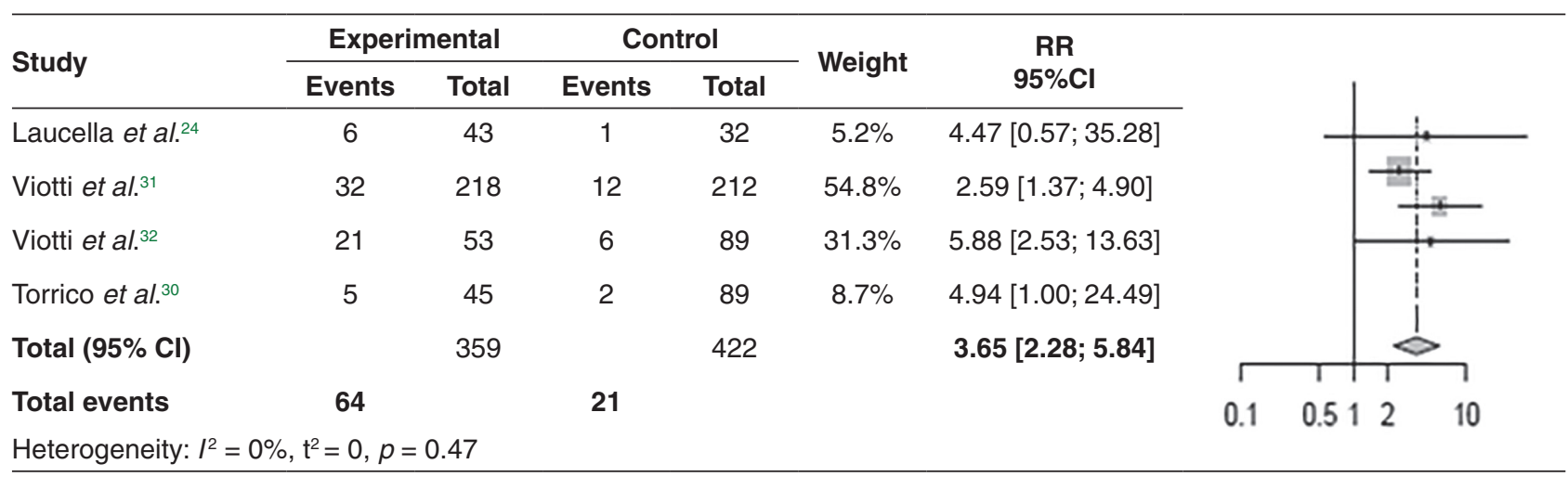

follow-up periods varied widely, from six months to 9.8 years. It is worth mentioning that the follow-up time is an important variable since, as previously mentioned, there is no consensual marker for the cure of the disease. Its evolution takes time, and the specific antibody titers for T.cruzi take a long time to decrease. Therefore, longterm investigations are needed to detect reliable negative results ${ }^{8,36}$.

Besides the factors associated with cure, the studies that adopted 60 days of treatment have also presented worse quality and more methodological problems according to Downs and Black ${ }^{10}$. Furthermore, the clinical heterogeneity found was high. The heterogeneity in the meta-analysis describes the difference among the treatment effects, which means that these differences are not random. The differences among the studies are related to the sample size, time of follow up, and different study protocol ${ }^{37}$. Because of this heterogeneity, although our results pointed out that 60 days of treatment does not seem beneficial for patients with Chagas disease in the chronic phase, more studies are necessary.

Previous studies pointed out that a long treatment presents more adverse effects ${ }^{38}$. According to Aldasoro et $a l .^{38}$, there is a high frequency of adverse effects for the dose of $5 \mathrm{mg} / \mathrm{kg} /$ day when used for 60 days, with a significant disease burden for patients. In a retrospective study in which $23.2 \%$ of treatment interruption was observed, a multivariate analysis showed that this interruption was significantly associated with the severity of adverse effects ${ }^{39}$.

In the study of Álvarez et al. ${ }^{6}$, no serious adverse effects were reported and in only one case the treatment was discontinued. However, the standard dose was administered through a new intermittent dosing regimen. This has emerged as a potential therapeutic scheme for the treatment of patients with Chagas disease in the chronic phase, but it has not yet be confirmed by further long-term evaluations ${ }^{6,8}$. Fabbro et al. $^{23}$ have also administere the
$5 \mathrm{mg} / \mathrm{kg} /$ day dose for 30 days, but only half the dose during the first week. However, no advantages were observed with the dose reduction only at the beginning of the treatment.

Two studies further evaluated the standard dose of benznidazole combined with another drug. Combination therapy has also been gaining attention as a possible strategy, since the association of molecules with different mechanisms of action could improve the pharmacokinetic profile, reducing the required drug dose, and increasing the treatment efficacy ${ }^{40}$. However, in STOP-CHAGAS, the combination of benznidazole and posaconazole showed no additional benefits compared with benznidazole in monotherapy, as both had similar negative PCR rates ${ }^{27}$. In the study by Perez-Mazliah et al. ${ }^{17}$, in which a sequential combination of benznidazole and allopurinol was used, significant changes in $\mathrm{T}$ and $\mathrm{B}$ cell responses, indicating a reduction in the parasite load, were observed. Studies with this type of therapy in humans, however, are still scarce, and further investigation is needed to define a better treatment combination.

Fernándes et al. ${ }^{11}$ tested a lower dose, between $2.5-55 \mathrm{mg} / \mathrm{kg} /$ day for 60 days. Negative PCR results were found in $100.0 \%$ of the participants and no serious adverse effects were reported. Molina et al..$^{25}$ and Morillo et al. ${ }^{26}$, used $150 \mathrm{mg}$ regimens for 60 days and $300 \mathrm{mg}$ for 40 to 80 days respectively. However, even if they used half of the dose, there were serious adverse effects for both studies, which led to discontinuation of treatment. In addition, negative PCR results were in the $60.0 \%$ range. These findings reinforce the idea that higher doses as well as longterm treatment are not beneficial to patients.

Regarding the observed outcomes, it is known that in chronically infected patients, traditional parasitological methods are not as sensitive as in the acute phase and that T. cruzi-specific antibody titers take years to decrease ${ }^{8,36}$. Thus, PCR is the most widely used and is considered a promising technique to detect therapeutic failure or success $^{8}$. However, PCR was not used in all the studies, 
but many of them have combined two or more outcomes, allowing better evaluations.

Most authors pointed out as a limitation, the loss of participants during the treatment period. This loss is usually due to the high frequency of adverse effects reported by benznidazole users, which leads to treatment abandonment ${ }^{7}$. The short follow-up time was also cited as a limitation. As previously stated, long-term investigations are necessary to detect reliable negative results ${ }^{8,36}$. Finally, the small sample number was also reported as a limitation. This is because with a small sample it is more difficult to obtain statistical power to answer the proposed hypotheses ${ }^{29}$.

In view of the above, it could be seen that the optimal dose regimen of benznidazole in the chronic phase of Chagas disease is still a matter of discussion today. Considering the results obtained in the present study and while an ideal dose is not defined, the standard dose of $5 \mathrm{mg} / \mathrm{kg} / \mathrm{day}$ for 30 days seems to be the best option for the treatment of patients with Chagas disease in the chronic phase. However, investigations should be stimulated in order to reach a consensus on the best dose schedule for the chronic phase of the disease.

\section{AUTHORS' CONTRIBUTIONS}

Conception and design or analysis and interpretation of data: VPF, NSS, CS and CDLO; writing of the article or relevant critical review of intellectual content: VPF, CS and CDLO.

\section{FUNDING}

This study was financed in part by the Coordenação de Aperfeiçoamento de Pessoal de Nível Superior - Brasil (CAPES) - Finance Code 001.

\section{REFERENCES}

1. Pan American Health Organization. Chagas disease. [cited 2020 Jul 1]. Available from: https://www.paho. org/hq/index.php?option=com_topics \& view=article\&id $=10 \&$ Itemid $=40242$ \&lang $=$ en

2. Brasil. Ministério da Saúde. Secretaria de Vigilância em Saúde. Coordenação-Geral de Desenvolvimento da Epidemiologia em Serviços. Guia de vigilância em saúde: volume único. $3^{\mathrm{a}}$ ed. Brasília: Ministério da Saúde; 2019. [cited 2020 Jul 1]. Available from: http://saude.gov.br/images/pdf/2019/junho/25/ guia-vigilancia-saude-volume-unico-3ed.pdf

3. Brasil. Ministério da Saúde. Comissão Nacional de Incorporação de Tecnologias no SUS. Protocolo clínico e diretrizes terapêuticas Doença de Chagas: relatório de recomendação. [cited 2020 Jul 1]. Available from: http://conitec.gov.br/images/ Relatorios/2018/Recomendacao/Relatorio_PCDT_Doenca_ de_Chagas.pdf

4. Soy D, Aldasoro E, Guerrero L, Posada E, Serret N, Mejía $\mathrm{T}$, et al. Population pharmacokinetics of benznidazole in adult patients with Chagas disease. Antimicrob Agents Chemother. 2015;59:3342-9.

5. Bermudez J, Davies C, Simonazzi A, Real JP, Palma S. Current drug therapy and pharmaceutical challenges for Chagas disease. Acta Trop. 2016;156:1-16.

6. Álvarez MG, Hernández Y, Bertocchi G, Fernández M, Lococo B, Ramírez JC, et al. New scheme of intermittent benznidazole administration in patients chronically infected with Trypanossoma cruzi: a pilot short-term follow-up study with adult patients. Antimicrob Agents Chemother. 2016;60:833-7.

7. Oliveira MJ, Forja JA, Oliveira AJ. Therapeutic drug monitoring of benznidazole and nifurtimox: a systematic review and quality assessment of published clinical practice guidelines. Rev Soc Bras Med Trop. 2017;50:748-55.

8. Kratz JM, Bournissen FG, Forsyth CJ, Sosa-Estani S. Clinical and pharmacological profile for treatment of Chagas disease. Expert Rev Clin Pharmacol. 2018;11:943-57.

9. Moher D, Liberati A, Tetzlaff J, Altman DG. Preferred reporting items for systematic reviews and meta- analyses: the PRISMA statement. Ann Intern Med. 2009;151:264-9.

10. Downs SH, Black N. The feasibility of creating a checklist for the assessment of the methodological quality both of randomised and non-randomised studies of health care interventions. J Epidemiol Community Health. 1998;52:377-84.

11. Fernández ML, Marson ME, Ramirez JC, Mastrantonio G, Schijman AG, Altcheh J, et al. Pharmacokinetic and pharmacodynamic responses in adult patients with Chagas disease treated with a new formulation of benznidazole. Mem Inst Oswaldo Cruz. 2016; 111:218-21.

12. Andrade MC, Oliveira MF, Nagao-Dias AT, Coêlho IC, Cândido DS, Freitas EF, et al. Clinical and serological evolution in chronic Chagas disease patients in a 4-year pharmacotherapy follow-up: a preliminary study. Rev Soc Bras Med Trop. 2013;46:776-8.

13. Andrade MC, Rocha EA, Costa AC, Lima DA, Silva Filho JD, Bezerra FS, et al. Evolução eletrocardiográfica e sorológica de pacientes com doença de Chagas crônica acompanhados por seis anos após o tratamento com benzonidazol. Relampa. 2016;29:141-9.

14. Lana M, Lopes LA, Martins HR, Bahia MT, Machado-de-Assis JM, Wendling AP, et al. Clinical and laboratory status of patients with chronic Chagas disease living in a vectorcontrolled area in Minas Gerais, Brazil, before and nine years after aetiological treatment. Mem Inst Oswaldo Cruz. 2009;104:1139-47. 
15. Levi GC, Lobo IM, Kallás EG, Amato Neto V. Etiological drug treatment of human infection by Trypanosoma cruzi. Rev Inst Med Trop Sao Paulo. 1996;38:35-8.

16. Oliveira LF, Silva VJ, Lages-Silva E, Molina RJ, Fuzissaki J, Niederhaur $\mathrm{S}$, et al. Heart rate variability in chronic Chagas patients before and after treatment with benznidazole. Auton Neurosci. 2010;158:118-22.

17. Perez-Mazliah DE, Álvarez MG, Cooley G, Lococo BE, Bertocchi G, Petti M, et al. Sequential combined treatment with allopurinol and benznidazole in the chronic phase of Trypanosoma cruziinfection: a pilot study. J Antimicrob Chemother. 2013;68:424-37.

18. Vallejo A, Monge-Maillo B, Gutiérrez C, Norman FF, LópezVélez R, Pérez-Molina J. Changes in the imune response after treatment with benznidazole versus no treatment in patients with chronic indeterminate Chagas disease. Acta Trop. 2016;164:117-24.

19. Pérez-Antón E, Egui A, Thomas MC, Puerta CJ, González JM, Cuéllar A, et al. Impact of benznidazole treatment on the functional response of Trypanosoma cruzi antigen-specific $\mathrm{CD} 4{ }^{+} \mathrm{CD}^{+} \mathrm{T}$ cells in chronic Chagas disease patients. PLoS Negl Trop Dis. 2018;12:e006480.

20. Antunes AP, Ribeiro AL, Sabino EC, Silveira MF, Oliveira $\mathrm{CD}$, Botelho CC. Benznidazole therapy for Chagas disease in asymptomatic Trypanosoma cruzi-seropositive former blood donors: evaluation of the efficacy of different treatment regimens. Rev Soc Bras Med Trop. 2016;49:713-20.

21. Braga MS, Lauria-Pires L, Argañaraz ER, Nascimento RJ, Teixeira AR. Persistent infections in chronic Chaga's disease patients treated with anti-Trypanosoma cruzi nitroderivatives. Rev Inst Med Trop Sao Paulo. 2000;42:157-61.

22. Coura JR, Abreu LL, Willcox HP, Petana W. Estudo Comparativo controlado com emprego de benznidazole, nifurtimox e placebo, na forma crônica da Doença de Chagas, em uma área de campo com transmissão interrompida. I Avaliação Preliminar. Rev Soc Bras Med Trop. 1997;30:139-44.

23. Fabbro DL, Streiger ML, Arias ED, Bizai ML, del Barco M, Amicone NA. Trypanocide treatment among adult with chronic Chagas disease living in Santa Fe City (Argentina), over a mean follow-up of 21 years: parasitological, serological and clinical evolution. Rev Soc Bras Med Trop. 2007;40:1-10.

24. Laucella AS, Mazliah DP, Bertocchi G, Álvarez MG, Cooley G, Viotti R, et al. Changes in Trypanosoma cruzi-specific imune responses following treatment: surrogate markers of treatment efficacy. Clin Infect Dis. 2009;49:1675-84.

25. Molina I, Gómez i Prat J, Salvador F, Treviño B, Sulleiro E, Serre $\mathrm{N}$, et al. Randomized trial of posaconazole and benznidazole for chronic Chagas' disease. N Engl J Med. 2014;370:1899-908.

26. Morillo C, Marin-Neto JA, Avezum A, Sosa-Estani S, Rassi A, Rosas F, et al. Randomized trial of benznidazole for chronic Chagas' cardiomyopathy. N Engl J Med. 2015;373:1295-306.
27. Morillo C, Waskin H, Sosa-Estani S, Bangher M, Cuneo C, Milesi R, et al. Benznidazole and posaconazole in eliminating parasites in asymptomatic T. Cruzi carriers: the STOPCHAGAS Trial. J Am Coll Cardiol. 2017;69:939-47.

28. Pinazo MJ, Guerrero L, Posada E, Rodríguez E, Soy D, Gascon J. Benznidazole-related adverse drug reactions and their relationship to serum drug concentrations in patients with chronic Chagas disease. Antimicrob Agents Chemother. 2013;57:390-5.

29. Pinazo MJ, Posada EJ, Izquierdo L, Tassies D, Marques AF, Lazzari E, et al. Altered hypercoagulability factors in patients with chronic Chagas disease: potential biomarkers of therapeutic response. PLOS Negl Trop Dis. 2016;10:e0004269.

30. Torrico F, Gascon J, Ortiz L, Alonso-Vega C, Pinazo MJ, Schijman A, et al. Treatment of adult chronic indeterminate Chagas disease with benznidazole and three E1224 dosing regimens: a proof-of-concept, randomized, placebo-controlled trial. Lancet Infec Dis. 2018;18:419-30.

31. Viotti R, Vigliano C, Lococo B, Bertocchi G, Petti M, Álvarez $\mathrm{MG}$, et al. Long-term cardiac outcomes of treating chronic Chagas disease with benznidazole versus no treatment: a nonrandomized trial. Ann Intern Med. 2006;144:724-34.

32. Viotti R, Vigliano C, Álvarez MG, Lococo B, Petti M, Bertocchi $\mathrm{G}$, et al. Impact of aetiological treatment on conventional and multiplex serology in chronic Chagas disease. Plos Negl Trop Dis. 2011;5:e1314.

33. Vallejo A, Monge-Maillo B, Gutiérrez C, Norman FF, LópezVélez R, Pérez-Molina J. Changes in the imune response after treatment with benznidazole versus no treatment in patients with chronic indeterminate Chagas disease. Acta Trop. 2016;164:117-24.

34. Lidani KC, Andrade FA, Bavia L, Damasceno FL, Beltrame $\mathrm{MH}$, Messias-Reason IJ. Chagas disease: from discovery to a worldwide health problem. Front Public Health. 2019;7:166.

35. Sulleiro E, Silgado A, Serre-Delcor N, Salvador F, Tavares de Oliveira M, Moure Z, et al. Usefulness of real-time PCR during follow-up of patients treated with Benznidazole for chronic Chagas disease: experience in two referral centers in Barcelona. PLoS Negl Trop Dis. 2020;14:e0008067.

36. Parrado R, Ramirez JC, de la Barra A, Alonso-Vega C, Juiz $\mathrm{N}$, Ortiz L, et al. Usefulness of serial blood sampling and PCR replicates for treatment monitoring of patients with chronic Chagas disease. Antimicrob Agents Chemother. 2019;63:e01191-18.

37. Barili F, Parolari A, Kappetein PA, Freemantle N. Statistical Primer: heterogeneity, random- or fixed-effects model analyses? Interact Cardiovasc Thorac Surg. 2018;27:317-21.

38. Aldasoro E, Posada E, Requena-Méndez A, Calvo-Cano A, Serret $\mathrm{N}$, Caselhas A, et al. What to expect and when: benznidazole toxicity in chronic Chagas' disease treatment. J Antimicrob Chemother. 2018;73:1060-7. 
39. Olivera MJ, Cucunubá ZM, Valencia-Hernández, Herazo R, Agreda-Rudenko D, Flórez C, et al. Risk factors for treatment interruption and severe adverse effects to benznidazole in adult patients with Chagas disease. PLoS One. 2017;12:e0185033.
40. Aguilera E, Alvarez G, Cerecetto H, González M. Polypharmacology in the treatment of Chagas disease. Curr Med Chem. 2019;26:4476-89.

\section{ERRATUM}

Page 1, where it reads:

Nayara Ragi Baldoni Couto

Should be read:

Nayara Ragi Baldoni

Page 11, where it reads:

\section{FUNDING}

This study was financed in part by the Coordenação de Aperfeiçoamento de Pessoal de Nível Superior - Brasil (CAPES) - Finance Code 001.

\section{Should be read:}

\section{FUNDING}

This study was financed in part by the Coordenação de Aperfeiçoamento de Pessoal de Nível Superior (CAPES), Finance Code 001, and Conselho Nacional de Desenvolvimento Científico e Tecnológico (CNPq), grant $\mathrm{N}^{\mathrm{o}} 421763 / 2018-3$.

http://dx.doi.org/10.1590/s1678-9946202062052 